\title{
Novel Hemostatic Factor Levels and Risk of Ischemic Stroke: The Atherosclerosis Risk in Communities (ARIC) Study
}

\author{
M. Fareed K. Suria Kazumasa Yamagishib, e Nena Aleksic ${ }^{\text {c, d }}$ Peter J. Hannan ${ }^{b}$ \\ Aaron R. Folsom ${ }^{\text {b }}$ \\ ${ }^{a}$ Department of Neurology, School of Medicine, and ${ }^{b}$ Division of Epidemiology and Community Health, \\ School of Public Health, University of Minnesota, Minneapolis, Minn., and 'Division of Hematology, Medical School \\ and ${ }^{\mathrm{d} D e p a r t m e n t}$ of Internal Medicine, University of Texas Health Science Center at Houston, Houston, Tex., USA; \\ e Department of Public Health Medicine, Graduate School of Comprehensive Human Sciences, and Institute of \\ Community Medicine, University of Tsukuba, Tsukuba, Japan
}

\section{Key Words}

Stroke $\cdot$ Coagulation $\cdot$ Risk factors

\begin{abstract}
Background and Objective: The role of hemostatic factor levels in cerebral infarction remains uncertain. We studied the association of levels of several under-studied hemostatic factors with ischemic stroke in a population-based cohort. Methods: The Atherosclerosis Risk in Communities (ARIC) study includes 15,792 individuals aged 45-54 years at intake. Hemostatic factors II, V, IX, X, XI, XII, plasminogen and $\alpha_{2^{-}}$ antiplasmin were measured on frozen citrate plasma samples from 1990 to 1992. A case-cohort design was used, including all incident ischemic strokes $(n=89)$ over a median of 7.5 years and a stratified cohort random sample $(n=412)$. To determine the association of hemostatic factors with incident ischemic stroke, we computed hazard ratios (HRs) using multivariate proportional hazard regression analyses adjusted for demographic and other cardiovascular risk factors. Results: The cohort random sample had a mean age (SD) of 56.9 (5.4) years and $42 \%$ were men. The age-, sex- and raceadjusted HRs for highest versus lowest quartiles were: factor
\end{abstract}

XI (2.74, 95\% Cl 1.42-5.29), factor IX (1.92, 95\% Cl 0.99-3.73), and $\alpha_{2}$-antiplasmin $(2.24,95 \% \mathrm{Cl} 1.16-4.33)$. Corresponding$\mathrm{ly}$, the HRs of ischemic stroke per SD increment of factors XI, IX, and $\alpha_{2}$-antiplasmin were $1.64,1.46$ and 1.52 , respectively (all $\mathrm{p}<0.05$ ). After multivariate adjustment including other clinical variables, the standardized HR remained significant for factor XI (1.50, 95\% Cl 1.10-2.05), but no other factor. Conclusion: A greater level of factor XI was associated with an increased risk of ischemic stroke. Higher factor XI levels might help identify patients at elevated ischemic stroke risk.

Copyright $\odot 2010$ S. Karger AG, Basel

\section{Introduction}

Prospective epidemiological studies have suggested certain hemostatic factors - including fibrinogen [1-6], von Willebrand factor antigen/factor VIII $[4,6]$ and tissue plasminogen activator antigen [4] - may be risk factors for ischemic stroke. An association of factor XI with stroke has been noted in some case-control studies $[7,8]$, but remains to be studied prospectively. Many other hemostatic factors have been linked with other thrombotic

\section{KARGER}

두 2010 S. Karger AG, Basel

Fax +41613061234 E-Mail karger@karger.ch www.karger.com
Aaron R. Folsom, MD

Division of Epidemiology and Community Health, School of Public Health

University of Minnesota, 1300 South Second Street, Suite 300

Minneapolis, MN 55454 (USA)

E-Mail folso001@umn.edu 
diseases [9-13], but not with ischemic stroke. We prospectively studied the relationship of ischemic stroke incidence with plasma levels of factor II, V, IX, X, XI, XII, plasminogen and $\alpha_{2}$-antiplasmin in the Atherosclerosis Risk in Communities (ARIC) study.

\section{Methods}

\section{Study Population}

The ARIC study includes a population-based cohort of 15,792 individuals aged 45-64 years at baseline in 1987 through 1989. The individuals were a probability sample of households from Jackson, Miss. (African-Americans only); Forsyth County, N.C.; Washington County, Md., and the northwestern suburbs of Minneapolis, Minn. Participants were reexamined at 3 follow-up visits: $1990-1992$ (93\% return rate), 1993-1995 (86\% return rate) and 1996-1998 (80\% return rate). The ARIC protocol was approved by the institutional review boards of the participating universities.

\section{Baseline Risk Factor Measurements}

After informed consent, seated blood pressure was measured 3 times after 5 -min rests and the mean of last 2 measurements was used for analysis. Total and high density lipoprotein cholesterol was measured using enzymatic methods. Use of antihypertensive medications within 2 weeks of each visit was recorded. Diabetes was defined as fasting serum glucose $\geq 126 \mathrm{mg} / \mathrm{dl}$, non-fasting glucose $\geq 200 \mathrm{mg} / \mathrm{dl}$, or physician-diagnosed or pharmacologically treated diabetes. The Cornell voltage algorithm [14] was used to define electrocardiographic left ventricular hypertrophy as score of $>28$ for men and $>22$ for women. Waist-hip ratio was calculated as the ratio of waist (umbilicus) to hip (maximum) girths. Smoking status (current, past or never) was obtained during interview.

\section{Ascertainment and Classification of Incident Stroke Cases}

The ARIC study followed the cohort by telephone interviews annually and by surveillance of hospital discharges and deaths in the ARIC communities. All hospitalized and fatal events were sought [15]. Medical records were abstracted and stroke events were classified as subarachnoid hemorrhage, intracerebral hemorrhage, thrombotic brain infarction or embolic brain infarction using standardized criteria including neurologic signs and imaging tests [16]. A sub-classification of definite or probable was used based on the level of certainty assessed using a predefined criteria. We defined ischemic stroke as a definite or probable thrombotic brain infarction or cardioembolic stroke.

\section{Case-Cohort Sample}

A case-cohort design was implemented at visit 2. Hemostatic factor levels of incident definite or probable ischemic stroke cases between ARIC visit 2 (1990-1992) and December 31, 1998 were compared with a stratified random sample of the ARIC cohort. Visit 2 was chosen as the baseline because the earliest blood samples available at the time of analysis were from visit 2 . Subjects were excluded if they had not participated in the visit 2 examination; if they had history of coronary heart disease or stroke at baseline or before visit 2; if they were African-American from the Washington
County or Minneapolis field center due to small numbers; or whose race was neither White nor African-American due to small numbers (total excluded $=3,014)$. An age- $(<55$ or $\geq 55$ years), gender- and race-stratified cohort random sample of 936 participants was selected from the eligible ARIC cohort $(\mathrm{n}=12,778)$. The number of White males sampled for the cohort random sample was equal to incident coronary heart disease cases; for the other racesex strata, the number of subjects sampled was twice the number of cases, to improve precision. From a combined list of incident stroke cases $(\mathrm{n}=248)$, incident coronary heart disease cases $(\mathrm{n}=$ $775)$ and the cohort random sample $(n=936)$, roughly half $(n=$ 969) of the subjects were randomly selected for measurement of hemostatic factors and successful hemostatic factor levels were obtained in 824 (85\%). This analysis therefore included 89 incident ischemic stroke cases and 412 cohort random sample subjects. Because of random sampling of the cohort, 6 cases with incident ischemic stroke were also in the cohort random sample.

\section{Laboratory Measures}

After identification of cases and noncases, citrate plasma samples frozen at $-70^{\circ} \mathrm{C}$ from visit 2 were retrieved and analyzed. Long-term stability of coagulation factors in plasma samples stored at $-70^{\circ} \mathrm{C}$ has been previously demonstrated [17]. Coagulation and chromogenic assays were performed on the ACL9000 instrument (Instrumentation Laboratory Co., Lexington, Mass., USA) according to the manufacturer's instructions. Coagulation factors II, V, IX, X, XI, and XII were measured using corresponding factor deficient plasmas and either a high sensitivity rabbit brain thromboplastin (IL Test PT-Fibrinogen HS) or aPTT reagent (rabbit brain phospholipids and silica; IL Test aPTT-C Activated Partial Thromboplastin Time). Plasminogen and $\alpha_{2}$-antiplasmin were measured by automated chromogenic assays (IL Test Plasminogen and ILTest plasmin inhibitor) using a synthetic chromogenic substrate S-2403. Calibration plasma (Assess Calibration Plasma, Instrumentation Laboratory) was used for the quantitation of coagulation and chromogenic tests, and standardized normal control plasma (Assess Normal Control, Instrumentation Laboratory) was used for the quality control.

To determine the reliability of field center processing plus laboratory assays, successive tubes were obtained from a single draw, and reliability coefficients of 46 replicate samples were calculated. Reliability coefficients of factors were 0.75 for II, 0.68 for V, 0.68 for IX, 0.82 for X, 0.90 for XI, 0.89 for XII, 0.86 for plasminogen and 0.68 for $\alpha_{2}$-antiplasmin.

\section{Statistical Analysis}

All analyses were weighted to take into account the stratified case-cohort sampling design [18]. Means and SDs of continuous hemostatic factors were calculated for ischemic stroke cases and noncases (weighted SDs) and compared using analysis of covariance (ANCOVA). Hazard ratios (HRs) and 95\% CI of incident ischemic stroke in relation to the baseline variables were computed by proportional hazards regression. First, hemostatic factor levels were used as continuous variables to compute standardized hazard ratios (i.e. per 1 SD increment). Then, hemostatic factor levels were divided into quartiles and regression models were created to determine the HRs and 95\% CIs for the second, third and highest quartiles, with the lowest quartile as the reference. Linear trend was tested by assigning median values of each analyte according to quartiles. Both models were first adjusted for age (continuous), 
sex and race (Black, White) and then also for systolic blood pressure (continuous), antihypertensive medication use (yes/no), total cholesterol (continuous), high density lipoprotein cholesterol (continuous), diabetes (yes/no), left ventricular hypertrophy on ECG (yes/no), waist-hip ratio (continuous) and smoking status (current, former, never). Additional models considered the effect of adjusting for hemostatic factors measured at ARIC visit 1: fibrinogen, factor VIIc, factor VIIIc and von Willebrand factor [6].

\section{Results}

At baseline the mean age of the cohort random sample was 56.9 years, $42 \%$ were men and $24 \%$ African-American. Over a median follow-up of 7.5 years, there were 89 incident ischemic stroke events. The age, race and sexadjusted mean hemostatic factor levels were not statistically different between incident ischemic stroke cases and the cohort random sample (table 1).

In proportional hazard analyses of each hemostatic factor, adjusted for age, sex, and race, factor IX, XI and $\alpha_{2}$-antiplasmin were positively and significantly associated (for trend, $\mathrm{p}<0.05$ ) with ischemic stroke incidence (model 1, table 2). The HR for the highest quartile was 1.92 for factor IX, 2.74 for factor XI and 2.24 for $\alpha_{2}$-antiplasmin when compared to the lowest quartile. Likewise, the respective HRs per SD increment was 1.46 for factor IX, 1.64 for factor XI and 1.52 for $\alpha_{2}$-antiplasmin. After adjustment for other major cardiovascular risk factors (model 2, table 2), the adjusted HR of ischemic stroke was 1.50 (1.10-2.05) per SD increment of factor XI.

There was a significant but weak correlation between factor XI and visit 1 measured plasma levels of fibrinogen (Spearman correlation coefficient $\mathrm{r}=0.12, \mathrm{p}=0.015)$, VII $(\mathrm{r}=0.12, \mathrm{p}=0.014)$, factor VIII $(\mathrm{r}=0.31, \mathrm{p}<0.001), \mathrm{vWF}$ $(\mathrm{r}=0.22, \mathrm{p}<0.001)$. Inclusion of these variables in regression model 2 did not change the HR for factor XI.

To further understand the confounding of associations by other cardiovascular risk factors, we determined the correlates of factor XI in the cohort random sample (table 3). Compared to higher quartiles, participants with factor XI levels in lowest quartile were more likely to be male, Caucasian, not have diabetes and to have lower total cholesterol. Similar analyses for factor IX (not shown) demonstrated that participants in the highest quartile compared to lowest quartile were more likely to be African-American (31 vs. 17\%) and diabetic (34 vs. 7\%). Participants in highest quartile of $\alpha_{2}$-antiplasmin were less likely to be male ( 25 vs. $53 \%$ ) and more likely to be diabetic ( 22 vs. $10 \%$ ) and on antihypertensive medication (43 vs. $22 \%$ ) (data not shown).

Hemostatic Factors and Ischemic Stroke
Table 1. Unadjusted mean values of hemostatic factors

\begin{tabular}{llrl}
\hline & $\begin{array}{l}\text { Incident ischemic } \\
\text { stroke } \\
(\mathrm{n}=89)\end{array}$ & $\begin{array}{l}\text { Non-cases } \\
(\mathrm{n}=406)^{1}\end{array}$ & $\begin{array}{l}\mathrm{p} \\
\text { value }^{2}\end{array}$ \\
\hline Factor II, \% & $101.3 \pm 17.2$ & $99.6 \pm 11.9$ & 0.70 \\
Factor V, \% & $112.1 \pm 31.9$ & $105.2 \pm 33.7$ & 0.57 \\
Factor IX, \% & $100.8 \pm 35.1$ & $88.2 \pm 28.2$ & 0.22 \\
Factor X, \% & $102.4 \pm 23.4$ & $102.8 \pm 25.7$ & 0.97 \\
Factor XI, \% & $93.0 \pm 24.2$ & $83.8 \pm 19.2$ & 0.18 \\
Factor XII, \% & $73.0 \pm 25.4$ & $78.7 \pm 23.9$ & 0.51 \\
$\alpha_{2}$-Antiplasmin, \% & $100.7 \pm 10.1$ & $97.6 \pm 13.0$ & 0.51 \\
Plasminogen, \% & $117.6 \pm 14.5$ & $116.0 \pm 13.9$ & 0.75 \\
\hline
\end{tabular}

${ }^{1}$ Unweighted SDs presented to allow comparison with cases.

${ }^{2}$ ANCOVA adjusted for age, sex and race.

\section{Discussion}

In this prospective study, individuals with greater levels of factor XI had a higher risk of ischemic stroke than those with low levels of factor XI. This was independent of other cardiovascular and important hemostatic factors, including vWF, factor VIII and fibrinogen. An increased risk of stroke was also noted with elevated levels of $\alpha_{2}$-antiplasmin and factor IX, but was not independent of other cardiovascular risk factors. We did not identify any relationship between factors II, V, XII, X and plasminogen levels and ischemic stroke.

Factor XI is traditionally classified as a member of the intrinsic pathway of coagulation. It is activated by factor XII, and increases thrombin generation through intermediary activation of factor IX and X. However, recent discoveries have suggested a more central and prothrombotic role of factor XI, in that thrombin may be a potent activator of factor XI [19]. This thrombin-mediated factor $\mathrm{XI}$ activation creates a feedback loop of thrombin generation, resulting in formation of more fibrin [20]. Increased thrombin generation also activates thrombin-activatable fibrinolysis inhibitor, further stabilizing the fibrin clot [21].

Case-control studies have noted an association of elevated levels of factor XI with deep venous thrombosis and myocardial infarction [10, 22]. Yang et al. reported that factor XI levels were higher in patients with ischemic stroke, transient ischemic attack or venous thrombosis than in healthy controls [7]. Recently, Santamaria et al. [8] studied factor XI levels in 218 patients with ischemic stroke and 227 age-, sex- and ethnicity-matched controls.

Cerebrovasc Dis 2010;29:497-502 
Table 2. Adjusted HR and 95\% CI for incident ischemic stroke across quartiles of hemostasis variables and per SD increment

\begin{tabular}{|c|c|c|c|c|c|c|c|}
\hline & & 1st quartile & 2nd quartile & 3rd quartile & 4th quartile & $\mathrm{p}$ for trend ${ }^{1}$ & HR per SD \\
\hline \multirow{3}{*}{$\begin{array}{l}\text { Factor II, \% } \\
(1 \mathrm{SD}=11.9)\end{array}$} & range & $61.1-92.2$ & $>92.2-98.0$ & $>98.0-105.0$ & $>105-159.8$ & & \\
\hline & model 1 & 1.0 & $1.42(0.74-2.70)$ & $0.66(0.30-1.46)$ & $1.31(0.67-2.58)$ & 0.70 & $1.08(0.82-1.41)$ \\
\hline & model 2 & 1.0 & $1.40(0.58-3.36)$ & $0.79(0.30-2.03)$ & $1.34(0.51-3.48)$ & 0.78 & $1.04(0.72-1.49)$ \\
\hline \multirow{3}{*}{$\begin{array}{l}\text { Factor V, \% } \\
(1 \mathrm{SD}=33.6)\end{array}$} & range & $13.3-83.5$ & $>83.5-109.0$ & $>109.0-128.0$ & $>128.0-202$ & & \\
\hline & model 1 & 1.0 & $1.75(0.85-3.59)$ & $1.63(0.79-3.38)$ & $1.77(0.86-3.65)$ & 0.12 & $1.25(0.98-1.60)$ \\
\hline & model 2 & 1.0 & $1.31(0.56-3.08)$ & $1.43(0.59-3.44)$ & $1.59(0.68-3.76)$ & 0.27 & $1.21(0.89-1.66)$ \\
\hline \multirow{3}{*}{$\begin{array}{l}\text { Factor IX, \% } \\
(1 \mathrm{SD}=29.3)\end{array}$} & range & $23.1-71.4$ & $>71.4-87.0$ & $>87.0-104.0$ & $>104.0-250$ & & \\
\hline & model 1 & 1.0 & $0.94(0.45-1.98)$ & $1.30(0.64-2.62)$ & $1.92(0.99-3.73)$ & 0.03 & $1.46(1.16-1.84)$ \\
\hline & model 2 & 1.0 & $0.82(0.36-1.87)$ & $0.62(0.26-1.50)$ & $0.96(0.42-2.19)$ & 0.95 & $1.16(0.86-1.56)$ \\
\hline \multirow{3}{*}{$\begin{array}{l}\text { Factor X, \% } \\
(1 \mathrm{SD}=25.8)\end{array}$} & range & $43.0-85.6$ & $>85.6-99.5$ & $>99.5-112.0$ & $>112.0-266.0$ & & \\
\hline & model 1 & 1.0 & $1.05(0.55-2.02)$ & $0.75(0.37-1.54)$ & $1.45(0.78-2.70)$ & 0.34 & $1.07(0.87-1.32)$ \\
\hline & model 2 & 1.0 & $0.75(0.32-1.78)$ & $0.52(0.21-1.28)$ & $0.88(0.38-2.05)$ & 0.79 & $0.97(0.70-1.33)$ \\
\hline \multirow{3}{*}{$\begin{array}{l}\text { Factor XI, \% } \\
(1 \mathrm{SD}=19.7)\end{array}$} & range & $36.1-69.1$ & $>69.1-82.3$ & $>82.3-95.7$ & $>95.7-182.0$ & & \\
\hline & model 1 & 1.0 & $1.00(0.47-2.11)$ & $1.18(0.56-2.51)$ & $2.74(1.42-5.29)$ & 0.001 & $1.64(1.27-2.11)$ \\
\hline & model 2 & 1.0 & $0.76(0.31-1.88)$ & $0.96(0.38-2.40)$ & $1.62(0.74-3.55)$ & 0.095 & $1.50(1.10-2.05)$ \\
\hline \multirow{3}{*}{$\begin{array}{l}\text { Factor XII, \% } \\
(1 \mathrm{SD}=24.0)\end{array}$} & range & $20.4-61.4$ & $>61.4-77.4$ & $>77.4-91.9$ & $>91.9-157.0$ & & \\
\hline & model 1 & 1.0 & $0.65(0.35-1.23)$ & $0.47(0.23-0.98)$ & $0.76(0.40-1.42)$ & 0.26 & $0.90(0.69-1.16)$ \\
\hline & model 2 & 1.0 & $0.87(0.40-1.90)$ & $0.60(0.25-1.45)$ & $1.16(0.52-2.61)$ & 0.99 & $1.00(0.72-1.38)$ \\
\hline \multirow{3}{*}{$\begin{array}{l}\alpha_{2} \text {-Antiplasmin, } \% \\
(1 \mathrm{SD}=12.9)\end{array}$} & range & $35.3-91.3$ & $>91.3-98.4$ & $>98.4-105.0$ & $>105.0-135.5$ & & \\
\hline & model 1 & 1.0 & $1.16(0.58-2.31)$ & $1.00(0.48-2.05)$ & $2.24(1.16-4.33)$ & 0.03 & $1.52(1.17-1.97)$ \\
\hline & model 2 & 1.0 & $1.14(0.50-2.60)$ & $0.86(0.37-1.99)$ & $1.36(0.57-3.24)$ & 0.56 & $1.21(0.92-1.58)$ \\
\hline \multirow{3}{*}{$\begin{array}{l}\text { Plasminogen, } \% \\
(1 \mathrm{SD}=14.0)\end{array}$} & range & $71.4-105.8$ & $105.8-116.0$ & $>116.0-125.0$ & $>125.0-158.5$ & & \\
\hline & model 1 & 1.0 & $1.30(0.65-2.58)$ & $1.11(0.52-2.35)$ & $1.69(0.84-3.41)$ & 0.19 & $1.20(0.93-1.56)$ \\
\hline & model 2 & 1.0 & $1.05(0.46-2.35)$ & $1.14(0.47-2.81)$ & $1.20(0.50-2.88)$ & 0.65 & $1.04(0.75-1.44)$ \\
\hline
\end{tabular}

Model 1: adjusted for age, sex and race.

Model 2: further adjusted for systolic blood pressure, antihypertensive medication use, total and high density lipoprotein cholesterol, diabetes, left ventricular hypertrophy, waist-hip ratio, and smoking status. Subjects with missing covariates were eliminated from model 2 analyses.

${ }^{1}$ Test for trend in HRs across quartiles.

Table 3. Visit 2 demographic and clinical characteristics by quartiles of factor XI

\begin{tabular}{lcccc}
\hline & $\begin{array}{c}\text { 1st quartile } \\
(36.1-69.1 \%)\end{array}$ & $\begin{array}{c}\text { 2nd quartile } \\
(>69.1-82.3 \%)\end{array}$ & $\begin{array}{c}\text { 3rd quartile } \\
(>82.3-95.7 \%)\end{array}$ & $\begin{array}{c}4 \text { th quartile } \\
(>95.7-182.0 \%)\end{array}$ \\
\hline $\mathrm{n}$ & 101 & 105 & 99 & 107 \\
Age, years & $56.2 \pm 5.5$ & $57.2 \pm 5.4$ & $57.0 \pm 5.4$ & $57.1 \pm 5.1$ \\
Male, \% & 54 & 42 & 37 & 35 \\
African-American, \% & 24 & 18 & 24 & 31 \\
Systolic blood pressure, mm Hg & $118 \pm 18$ & $121 \pm 19$ & $123 \pm 17$ & $23 \pm 20$ \\
Antihypertensive medication use, \% & 31 & 28 & $212 \pm 39$ & 37 \\
Total cholesterol, mg/dl & $194 \pm 34$ & $210 \pm 37$ & $50 \pm 16$ & $210 \pm 41$ \\
HDL cholesterol, mg/dl & $50 \pm 18$ & $53 \pm 16$ & 14 & $48 \pm 16$ \\
Diabetes, \% & 10 & 10 & 2 & 30 \\
ECG, left ventricular hypertrophy, \% & 2 & 3 & 2 \\
Waist-hip ratio & $0.92 \pm 0.08$ & $0.91 \pm 0.08$ & $0.92 \pm 0.08$ & 11 \\
Current smoker, \% & 18 & 21 & $0.92 \pm 0.07$ \\
\hline
\end{tabular}

Data presented as means \pm SD or percentages. 
A high factor XI level was strongly associated with ischemic stroke with an odds ratio of 6.4, but only in dyslipidemic subjects. Due to the case-control design, causal relationships of high factor XI with thrombosis could not be established from these studies. The prospective design of our study helps establish a causal relationship.

Factor XI has been noted to be essential for thrombosis caused by $\mathrm{FeCl}_{3}$-induced injury to vessel walls in mice [23]. Renne et al. [24] studied vessel occlusion by mechanical aortic injury in wild-type, factor-XII-deficient and factor-XI-deficient mice. Whereas, the vessel completely and irreversibly occluded in wild-type mice, the occlusion was delayed and reversible in both factor-XII- and factor-XI-deficient mice. Kleinschnitz et al. [25] reported diminished brain infarct volumes after transient occlusion of the middle cerebral artery in factor-XI-deficient mice compared to wild-type mice. These findings suggest an important role of factor XI in thrombus propagation. Recently, Salomon et al. [26] noted the incidence of ischemic stroke was significantly lower than expected in individuals with factor XI deficiency. We found an increased risk of stroke in the highest quartile of factor XI levels. It is possible that this is because of reduced thrombosis in subjects with lower factor XI levels.

High factor IX levels have been associated with an increased risk of venous thrombosis [13], but potential linkage to arterial thrombosis has not been studied. The association of arterial or venous thrombosis with $\alpha_{2}$-antiplasmin levels also has not been studied. We identified positive relations of factor XI and $\alpha_{2}$-antiplasmin with ischemic stroke after adjustment for demographic variables, but this was no longer significant after adjustment for other cardiovascular risk factors. This suggests confounding by other cardiovascular risk factors.
Some methodological issues regarding this study warrant discussion. The sample size was relatively small, so it is possible we missed some true association between hemostatic factors and ischemic stroke. It is also possible that a relation existed at extremely high or low factor levels but was not detectable with our small sample size. There was no association between factor XI levels and ischemic stroke in the ANCOVA analysis (table 1), but there was significant relationship in the regression analysis. Sensitivity analysis indicated that this was because ischemic stroke events in the higher factor XI quartiles occurred early during follow-up. The implications of this finding are unclear. Ischemic strokes of all subtypes are included in this study. It is possible that hemostatic factor levels are only important for certain ischemic stroke subtypes, but a very large study would be required to demonstrate this. The study population was relatively young for stroke outcome, and the association of hemostatic factors with stroke may differ in an elderly population.

In conclusion, this study identifies higher factor XI levels as a new potential marker for increased risk of ischemic stroke.

\section{Acknowledgments}

The authors thank the staff and participants of the ARIC study for their important contributions.

The ARIC study is supported by the National Heart, Lung, and Blood Institute (contracts N01-HC-55015, 55016, 55018, 55019, 55020, 55021 and 55022). K.Y. was supported by the Kanae Foundation for the Promotion of Medical Science, Tokyo, Japan. M.F.K.S. was supported by NIH 5K12-RR023247-03.

\section{References}

1 Wilhelmsen L, Svardsudd K, Korsan-Bengtsen $\mathrm{K}$, et al: Fibrinogen as a risk factor for stroke and myocardial infarction. N Engl J Med 1984;311:501-505.

$\checkmark 2$ Meade TW, Mellows S, Brozovic M, et al: Haemostatic function and ischaemic heart disease: principal results of the Northwick Park Heart Study. Lancet 1986;2:533-537.

-3 Kannel WB, Wolf PA, Castelli WP, D’Agostino RB: Fibrinogen and risk of cardiovascular disease: The Framingham Study. JAMA 1987;258:1183-1186.

\footnotetext{
4 Smith FB, Lee AJ, Fowkes FG, et al: Hemostatic factors as predictors of ischemic heart disease and stroke in the Edinburgh Artery Study. Arterioscler Thromb Vasc Biol 1997; 17:3321-3325.

5 Resch KL, Ernst E, Matrai A, Paulsen HF: Fibrinogen and viscosity as risk factors for subsequent cardiovascular events in stroke survivors. Ann Intern Med 1992;117:371-375.

6 Folsom AR, Rosamond WD, Shahar E, et al: Prospective study of markers of hemostatic function with risk of ischemic stroke. The Atherosclerosis Risk in Communities (ARIC) Study Investigators. Circulation 1999;100:736-742.
}

\footnotetext{
7 Yang DT, Flanders MM, Kim H, Rodgers GM: Elevated factor XI activity levels are associated with an increased odds ratio for cerebrovascular events. Am J Clin Pathol 2006;126:411-415

$\checkmark 8$ Santamaria A, Oliver A, Borrell M, et al: Higher risk of ischaemic stroke associated with factor XI levels in dyslipidaemic patients. Int J Clin Pract 2007;61:1819-1823.

$\checkmark 9$ Doggen CJ, Rosendaal FR, Meijers JC: Levels of intrinsic coagulation factors and the risk of myocardial infarction among men: opposite and synergistic effects of factors XI and XII. Blood 2006;108:4045-4051.
} 
-10 Meijers JC, Tekelenburg WL, Bouma BN, Bertina RM, Rosendaal FR: High levels of coagulation factor XI as a risk factor for venous thrombosis. N Engl J Med 2000;342:696701.

-11 de Visser MC, Poort SR, Vos HL, et al: Factor $\mathrm{X}$ levels, polymorphisms in the promoter region of factor $\mathrm{X}$, and the risk of venous thrombosis. Thromb Haemost 2001;85: 1011-1017.

-12 Poort SR, Rosendaal FR, Reitsma PH, Bertina RM: A common genetic variation in the 3 '-untranslated region of the prothrombin gene is associated with elevated plasma prothrombin levels and an increase in venous thrombosis. Blood 1996;88:3698-3703.

-13 van Hylckama Vlieg A, van der Linden IK, Bertina RM, Rosendaal FR: High levels of factor IX increase the risk of venous thrombosis. Blood 2000;95:3678-3682.

-14 Molloy TJ, Okin PM, Devereux RB, Kligfield P: Electrocardiographic detection of left ventricular hypertrophy by the simple QRS voltage-duration product. J Am Coll Cardiol 1992;20:1180-1186.

15 The Atherosclerosis Risk in Communities (ARIC) Study: design and objectives. The ARIC investigators. Am J Epidemiol 1989; 129:687-702.
6 Rosamond WD, Folsom AR, Chambless LE, et al: Stroke incidence and survival among middle-aged adults: 9-year follow-up of the Atherosclerosis Risk in Communities (ARIC) cohort. Stroke 1999;30:736-743.

17 Lewis MR, Callas PW, Jenny NS, Tracy RP Longitudinal stability of coagulation, fibrinolysis, and inflammation factors in stored plasma samples. Thromb Haemost 2001;86: 1495-1500.

18 Barlow WE: Robust variance estimation for the case-cohort design. Biometrics 1994;50: 1064-1072.

19 Baglia FA, Walsh PN: Thrombin-mediated feedback activation of factor XI on the activated platelet surface is preferred over contact activation by factor XIIa or factor XIa. J Biol Chem 2000;275:20514-20519.

20 Wielders SJ, Beguin S, Hemker HC, Lindhout T: Factor XI-dependent reciprocal thrombin generation consolidates blood coagulation when tissue factor is not available. Arterioscler Thromb Vasc Biol 2004;24: 1138-1142.

-21 Wang W, Boffa MB, Bajzar L, et al: A study of the mechanism of inhibition of fibrinolysis by activated thrombin-activable fibrinolysis inhibitor. J Biol Chem 1998;273:2717627181.
22 Merlo C, Wuillemin WA, Redondo M, et al: Elevated levels of plasma prekallikrein, high molecular weight kininogen and factor XI in coronary heart disease. Atherosclerosis 2002;161:261-267.

23 Rosen ED, Gailani D, Castellino FJ: FXI is essential for thrombus formation following $\mathrm{FeCl} 3$-induced injury of the carotid artery in the mouse. Thromb Haemost 2002;87:774776.

24 Renne T, Pozgajova M, Gruner S, et al: Defective thrombus formation in mice lacking coagulation factor XII. J Exp Med 2005;202: 271-281.

25 Kleinschnitz C, Stoll G, Bendszus M, et al: Targeting coagulation factor XII provides protection from pathological thrombosis in cerebral ischemia without interfering with hemostasis. J Exp Med 2006;203:513518.

26 Salomon O, Steinberg DM, Koren-Morag $\mathrm{N}$, Tanne D, Seligsohn U: Reduced incidence of ischemic stroke in patients with severe factor XI deficiency. Blood 2008;111: 4113-4117. 